\section{Zur Kritik und Ausgestaltung des Röntgenverfahrens.*)}

\section{Von Dr. Max Levy-Dorn in Berlin.}

Die Beweise von dem Werth der Röntgen'schen Strahlen für die Heilkunde und noch mehr von der Anerkennung dieses Werthes häuften sich im Verlauf des jetzigen Jahres zu einer Höhe, wie sie bisher kaum von einer anderen Untersuchungsmethode in so kurzer Zeit erreicht worden ist. Ein Krankenhaus nach dem andern schafft sich die Rontgenapparate an, die Zahl der Privatärzte, welche ein Gleiches thut, wächst beständig, und dies nicht allein hier in Deutschland, sondern auch in allen anderen Culturländern. Die meisten medicinischen Congresse beschäftigten sich eindringlich mit den X-Strahlen. Am grossartigsten aber ward ihre Wirkung auf dem XXVI. Congress der Deutschen Gesellschaft für Chirurgie zur Anschauung gebracht. Eine glänzende Ausstellung und ein ganzer Sitzungstag war ihnen gewidmet.

Die Litteratur über die Anwendung der nouen Methode ist bis zur Unübersichtlichkeit angewachsen; besondere Zeitungen wurden den Röntgenstrahlen gewidmet; ja in England hat sich eine Röntgengesellschaft gebildet!

Wenn von einer Sache viel die Rede ist, pflegen auch Vorschläge, ihren Namen zu ändern, nicht auszubleiben. So sind die Worte SkiaRadio-, Ixographie entstanden, welchen sich nun Pykno-, Elektro-, Diagraphie hinzugesellt haben. Einer, dem Röntgographie zu schlecht klang, empfiehlt dafür das musikalischere Wort „Rontographie“. Alle jene Ausdrücke werden gelegentlich gebraucht, obwohl sie mit Ausnahme des curiosen "Ixographie" nur immer eine Soite der nenen Kunst hervorheben oder zu allgemein gehalten sind. Meines Erachtens lässt sich gegen Röntg(en)ographie nicht mehr einwenden als gegen den entsprechenden, eingebürgerten Ausdruck „Daguerreotypie“.

Um nicht schon Gesagtes zu wiederholen, muss ich anf meine vorige Mittheilung in dieser Wochenschrift No. 8 verweisen, an welche sich auch das am Ende der Arbeit befindliche, systematisch geordnete Litteraturverzeichniss anschliesst. Vorher aber möchte ich gerade bei der Begeisterung, welche für die neue Methode herrscht, auf Irrthumer, die leicht durch sie entstehen können, hinweisen und dann die Grenzen angeben, bis zu welchen sie gelangt ist - soweit sich dies thun lässt, ohne sich zu sehr in Einzelheiten zu verlieren.

Beginnen wir mit dem sehr lehrreichen Fall von Lexer $\left.{ }^{40 * *}\right)$ : Eine Revolverkugel wird auf Grund der ersten Durchstrahlung in den Weichtheilen vor der ersten Rippe gesucht. Die Operation ist vergebens Später wird von verschiedenen Seiten durchstrahlt und auf der Haut der Punkt jedesmal bezeichnet, wo die Kugel erscheint. Aus der Combination der gesammten Beobachtungen ergiebt sich die wahre Lage des Geschosses, nämlich unterhalb des Processus transversus des ersten Brustwirbels, und die Entfernung der Kugel gelingt nun.

Berührt es den Operateur schon peinlich genug, wenn er aufs Ungewisse schneidet und sein Vorgehen ohne Erfolg bleibt, so demüthigt es ihn noch weit mehr, wenn er ein leicht erreichbares Ziel vor Augen zu haben glaubt, aber sein Messer vergebens arbeitet. Wen das Röntgenbild einmal gleichsam wie eine Fata morgana irregefuhtrt hat, der wird sich immer wieder und wieder fragen, durch welche Mittel er dem Mangel abhelfen kann, welche Methoden uns in bester Weise itber die Lage der innneren Theile in Kenntniss setzen. Die Grundsätze, nach welchen dies zu geschehen hat, sind schon längst festgesetzt. Ueber die in den verschiedenen Fällen zweckmässigste Form ihrer Anwendung sind in diesem Jahre einige neue Vorschläge gemacht worden.

Exner ${ }^{9}$ ) hat einen besonderen Apparat beschrieben, welcher es ermöglicht - allerdings mit Hülfe der Rechnung -, verhältnissmässig einfach auf dem Fluorescenzschirm Lage und Form von Fremdkörpern etc. zu bestimmen. I $\mathrm{ch}^{10}$ ) beschrieb ein sehr leichtes Verfahren, um in flachen Körpergegenden, wie die Hand, die in der Praxis nothwendigsten Ortsbestimmungen zu erlangen, gab eine Methode an, welche ohne Rechnung und Apparat durch einfache Construction, auch in umfassenderen Körpergegenden Lokalisationen zu machen erlaubt, und habe endlich die Stereoskopie für diesen Zweck verwerthet. Büttner und Müller ${ }^{2}$ ) schlagen vor, im Operationssaal Vorkehrungen für Durchstrahlungen zu treffen, um

*) Eine systematische Uebersicht der Litteratur ron Jannar bis Soptember findet sich am Ende der Arbeit.

**) Die Zahlen beziehen sich auf das Litteraturzeichniss. im Nothfall während der Operation untersuchen zu können. Aber keine Methode passt jedesmal. und es muss dem Tact des Untersuchers iberlassen bleiben, immer die zweckmässigste auszuwählen

Nicht weniger als bei der Ortsbestimmung von Fremdkorpern und dergleichen sind Versehen bei den Schlüssen aus Röntgenbildern auf die Gestalt und Form von Körpertheilen vorgekommen. So erwähnte König auf dem letzten Chirurgencongress, dass nach einem Bilde ein Fenurhals verkürzt erschien, der sich bei der zweiten Aufnahme nach Innenrotation des Schenkels als normal erwies. Oberst warnte ebendort, aus einem Bild auf eine Reposition von Knochen zu schliessen. Stechow betont, dass ein sehr sorgfältiger Vergleich der gesunden und kranken Seite in derselben Stellung nöthig ist, um geringe Veränderungen festzustellen.

Unkenntniss der Ossificationsverhältnisse veranlasste, wie Behrendsen ${ }^{52}$ ) berichtet, Bristow, einen doppelseitigen Defect der Patella bei einem zweijährigen Kinde auf Grund eines Rőntgogramms anzunehmen, obwohl in diesem Alter normaler Weise die Kniescheibe noch nicht verknöchert ist.

Selbst die leichteste Aufgabe, welche wir den X-Strahlen stellen können, nämlich zu bestimmen, ob ein metallischer Fremdkörper vorhanden ist, kann gelegentlich bei der Ausführung auf Schwierigkeiten stossen. Es kann $\mathrm{B}$ B ein Jodoformstreifen eine Nadel vortäuschen (Deutsche medicinische Wochenschrift S. 110) und andererseits ein dünnerer Fremdkörper "weggestrahlt" werden und dadurch dem Auge entgehen.

Unter Umständen können Plattenfehler zu Irrthümern Anlass geben, wie es mir einmal beim Aufsuchen von Schrotkörnern ergangen ist. Eine Wiederholung der Aufnahme bildet den Schutz davor.

Skiagramme auf gekrümmten Flächen, wie z. B. auf einem um ein Glied gelegtes photographisches Papier, geben sehr eigenthümliche und verrenkte Bilder, welche Missbildungen und dergleichen vortäuschen künnen. Ein solches Bild demonstrirte $\mathrm{Hedley}{ }^{14}$ ).

Fälle aus dem Gebiet der inneren Medicin, in welchen Fehldiagnosen auf Grund von Untersuchungen mit X-Strahlen gestellt wurden, sind sehr schwer zu sammelı, weil die Controlle des im Schattenbilde Gesehenen meist fehlt. Wenigstens ist es schwierig, den in Röntgen's Kunst weniger Bewanderten zu uberzeugen. Man lese z. B. die Discussion Schott-Dumstrey ${ }^{78-80}$ ). Ich möchte ausserdem auf die oft ausgesprochene, aber falsche Auffassung hinweisen, dass Pulsation im Schattenbild eines Tumors des Brustraums schlechterdings für Aortenaneurysma spreche. Wie ich bereits auf dem 15. Congress für innere Medicin hervorgehoben habe, pulsirt jede Geschwulst in der Nähe der grossen Gefässe selbstverständlich anch im Röntgenbilde. Aus der Art der rhythmischen Bewegung vermag man abor dennoch, wenn auch nicht immer sichere Schlüsse zu ziehen. Die von mir untersuchten Fälle von A ron ${ }^{83}$ ) und Grabow er ${ }^{96}$ ) mögen das Gesagte illustriren.

Nichtärzte, welche die neue Kunst treiben, sind noch ganz besonderen Gefahren ausgesetzt. Ich erinnere mich, dass mir jemand "Gallensteine" oberhalb des Zwerchfells zeigte! Ein anderer sah die normale Oberarmkrümmung im Schattenbilde für die Folge einer Fractur an.

Sehen wir uns nun die Grenzen an, innerhalb derer die Röntgenstrahlen Nutzen bringen. Sie sind nicht sehr viel weiter als voriges Jahr gesteckt worden; sie werden aber durch eine reiche Casuistik immer mehr und mehr gesicliert und genauer umschrieben.

In Bezug auf das Finden von Fremdkörpern seien zwei Grenzfälle aus meiner Praxis gegenübergestellt: In dem einen gelang es noch ein halbes Nähnadelöhr in der Handwurzel einer kräftigen, erwachsenen Frau zu entdecken, also einen Körper, welchen man frei daliegend wegen seiner Kleinheit nur mit Mühe finden würde; in dem anderen Falle vermochte ich nicht eine in den Rücken einer mageren Dame eingedrungene Năhnadel auf dem Schattenbilde zu erkennen. Im Gehirn konnte ich noch Schrotkörner, die nicht weit vom Scheitelhein lagen, sowohl nach horizontaler als verticaler Durchstrahlung auf der Platte fixiren. K ü m mell ${ }^{18}$ ) sah feine Drahtnägel, die sich ein Geisteskranker unter die Kopfhaut gesteckt hatte. Unter den Fremdkörpern des Abdomens spielt immer noch der Murphyknopf die Hauptrolle. Ich fand ausserdem nur noch, dass Butter und Mïller einen Nagel im Magen eines Kindes auf der Platte fixirt liaben.

In der Nïhe des Zwerchfells, wie in dem letzten Fall, aber auch im gauzen Brustraum werden infolge respiratorischer Verschiebung die Bilder von Fremdkörpern etc. leicht unscharf und eigenthümlich gestaltet. Es ist mir mit Hülfe von zwei Verstärkungsschirmen gelungen, die Aufnahme des Brustkorbes eines Erwachsenen klar und deutlich während einer Inspiration $\left(3_{4}\right.$ Minute) zustande zll bringen. Dadurch wird jener Uebelstand beseitigt.

In der Blase wurden wiederholt Haarnadeln mit Röntgenstrahlen festgestellt, siehe z. B. Wulstein ${ }^{20}$ ), Soiffart ${ }^{99}$ ). Des letzteren Mittheilung hat noch insofern besonderes Interesse, weil er die Methode mit Erfolg anwandte, bei der die für Röntgenstrahlen empfindliche Schicht (photographische Platte oder Fluorescenzschirm) in die Vagina eingeführt wird.

Das Skelett bietet der Röntgographie nur noch Schwierigkeit bei starken Erwachsenen und auch dort nur in der Gegend des Beckens und den schwer zugänglichen Theilen der Wirbelsäule (von der oberen 
Herzgrenze abwärts). Am Schädel sind Veränderungen unerheblicher Art immer noch kaum zu erkennen. Es gelang mir aber, bei einem jungen Manne eine kleine Vertiefung im Scheitelbein, welche nach einem Abscess zurückgeblieben war, schwach, aber dentlich auf der Platte als lichtere Stelle zum Ausdruck zu bringen. Oppenheim brachte ein Gehirn nebst Tumor in einen Schädel und bemerkte den Tumor auf dem Schattenbilde wieder.

Im ubrigen liegen zahlreiche Berichte ubber Läsionen, Geschwülste, plastische Operationen und Erkrankungen aller Art, selbst schwer zugänglicher Theile, wie des Hüftgelenks Erwachsener, vor, und ich könnte diese Mittheilungen leicht beträchtlich vermehren.

Die Veränderungen durch Osteomyelitis, von denen ich früher sagen musste, dass sie - am Menschen wenigstens - noch nicht durch XStrahlen erkannt werden können, sind nunmehr für sie wohl erreichbar. Sowohl die Sequester, wie die Abscesse markiren sich oftmals auf den Bildern, nicht selten aber besser auf dem Negativ, als auf dem Abzug. Man kann bei solchen Untersuchungen das Verfahren Becher's, bei Blutleere zu skiagraphiren, mit Vortheil verwenden.

Kleine (tuberkulöse) Heerde sind selbst noch an der Wirbelsäule auf Skiagrammen erkannt worden. Doch betreffen die Mittheilungen hierüber ausschliesslich Kinder. Gröbere Wirbelerkrankungen werden auch an Erwachsenen, besouders an den leichter zugänglichen Theilen nicht unverborgen bleiben.

Die Verkalkung der Gefüsse kann selbst bei tiefer Lage derselben zur Anschauung kommen, ob aber schon zu einer Zeit, in der sich die Arteriosklerose sonst durch nichts verräth, muss als zweifelhaft hingestellt werden.

Von den inneren Organen setzen immer noclı die des Abdomens der Untersuchung den grössten Widerstand entgegen. Man muss auch hier zwischen jugendlichen Personen and dünnen erwachsenen einerseits und dicken, besonders muskulösen Erwachsenen andererseits unterscheiden. Verhältnissmässig gute Bilder pflegen sehr grosse magere Individuen nach meinen Erfahrungen zu geben. Was gelegentlich auf dem Schattenbild im Abdomen deutlich differenzirt worden ist, betrifft (Fremdkörper ausgenommen) wohl aussch!iesslich Personen der erstgenannten Kategorie. Besonders geben Kinder ein relativ dankbares Material. K iimmell ${ }^{18}$ ) and Rosenfeld ${ }^{3}$ ) sahen gelegentlich an ihnen sogar die Schlingen des Dünndarms. Ich bemerkte auf Skiagrammen von Erwachsenen einige Male die Umrisse des Colon ascendens und descendens.

Die meisten Autoren - und ich muss mich ihnen anschliessen haben nur selten die Nieren deutlich, häufiger aber angedeutet gefunden. Wulstein ${ }^{20}$ ) und ebenso Rosenfeld ${ }^{3}$ ) berichten, dass sie dieselben oft auf ihren Bildern klar beobachteten.

Steine, besonders gut die Oxalate, können sowohl in der Niere als in der Blase photographirt werden. Nicht selten aber misslingt noch unser darauf gerichtetes Bemihen. Von der Sicherheit, welche die Diagnose der Fremdkörper und der meisten Knochenaffectionen bereits erreicht hat, ist die Erkennung von Steinen mit Hulfe der X-Strahlen noch weit entfernt. Das Skiagramm von Gallensteinen in situ ist hente nicht mehr als ein Curiosum.

Grunmach 23b) hat neuerdings die Hydronephrose eines 14 jährigen Knaben im Bilde ausgeprägt gefunden. Durch Metallsonden und Ein. bringen von Wismuthlösungen, haben verschiedene Autoren (Lindemann ${ }^{97}$ ), Wulstein ${ }^{20}$ ) die untere Magengrenze zur Anschauung gebracht. Bei starken Patienten ist, wie ich mich überzeugt habe, die Sonde vorzuziehen. Ebenso lässt sich ein Speiseröhrendivertikel durch die gleichen Mittel demonstriren.

Die Bewegungen des Gaumensegels kann man direkt auf dem Schirm verfolgen (Scheier ${ }^{94}$. Die Diagnose der Erkrankungen des Brustraums setzt grosse Erfahrung im Abschätzen der Schattentiefen und eine besondere Kenntniss der normalen Vertheilung der Schatten voraus. Man lasse sich durch einen ersten misslungenen Versuch nicht zurückschrecken. Durch Uebung lässt sich hier eine Sicherheit gewinnen, welche der eines tüchtigen Percusseurs mindestens gleichkommt. Um mich nicht zu sehr in Einzelheiten zu verlieren, muss ich in Bezug auf die übrigen Punkte auf meine fruhere Uebersicht und das Litteraturverzeichniss verweisen. In der Geburtshälfe und Gynäkologie beschränkt sich die Anwendung der Röntgenstrahlen auf Eruirung der Beckenformen (Wullstein $\left.{ }^{21}\right)$. Benedikt ${ }^{10+a}$ ), Levy und Thumin ${ }^{103}$ ) und auf die Möglichkeit, wenigstens den schon entwickelteren Fötus erkennen und seine Lago bestimmen zu können, wenngleich die Bilder noch unscharf werden (Levy-Dorn ${ }^{104}$ ).

Die für Röntgenaufnahmen nöthige Expositionszeit hat dieses Jahr schneller abgenommen, als wir uns träumen liessen. Sie zählt jetzt für Skiagramme der Hand nach Secunden, und sie beträgt für Becken Erwachsener drei bis fünf Minuten, selten mehr. Dieser Erfolg ist theils durch Verbesserung der Strahlenquelle, theils durch vollkommenere Ausnutzung der einmal gewonnenen Strahlen errungen worden. Die intensivsten Strahlen erhalten wir, wenn wir bei geeignetem Rohr bei nicht zn niedrigem Vacuum die sogenannte Antikathode zur Weissgluth bringen. Dieses Prinzip wurde zuerst von K ï mmell (Hamburg) in grossartigem Maassstabe angewandt.
Die bessere Ausnutzung der Strahlen geschieht theils durch dickere photographische Schichten $\left(\mathrm{C}_{0} \mathrm{wl}^{12}\right)$ oder in ähnlicher Weise durch Vermehrung derselben (Levy ${ }^{13}$ ), theils durch den gleichzeitigen Gebrauch fluorescirenderSchirme und photographischer Platten. Man bringt die fluorescirende Substanz des sogenannten Verstärkungsschirmes auf die photographisclie Schicht und bewirkt so, dass sich zı der direkten Finwirkung des Strahls anf die Platte diejenige des durch ihn hervorgerufenen Flnorescenzlichtes addirt. Solche Verstärkungsschirme sind seit langem empfohlen worden, waren aber entweder unzuverlässig oder nur für bestimmte Platten anwendbar oder gaben ein zu grobes Korn. Ihre Anwendung war daher nur im Nothfall zu empfehlen. Nenerdings hat mir nun die allgemeine Elektricitätsgesellschaft zu Berlin Schirme ïbersandt, in welchen die Uebelstände im wesentlichen beseitigt sind. Das Korn ist so gering, dass selbst die Knochenstructur auf den Bildern wiedererscheint. Doclı fällt diese dabei nicht immer tadellos aus, so dass derjenige, welcher derartige Stıdien, wie z. B. Gocht (Deutsche medicinische Wochenschrift No. 7) treiben will, noch gut daran thut, auf die Verstärkungsschirne zll verzichten. Fïr die Anforderung der Praxis dagegen empfiehlt sich jetzt -- besonders bei voluminöseren Körpertheilen - seine Anwendung sowohl im Interesse des Patienten, wie des Arztes. Ein Verstärknngssehirm verringert die Expositionszeit auf den vierten bis funften Theil.

Die Daner der Exposition darf bekanntlich ohne Schaden für den Patienten eine gewisse Zeit nicht itberschreiten. Diese Zeit ist kürzer bei angenähertem Rohr lind stärkeren Strahlen, im umgekehrten Falle grösser. Bei einer Intensität, welche die Mittelhandknochen noch in einer Entfernung von $3-4 \mathrm{~m}$ erkennen lässt, könnte man ohne Bedenken das Rohr ïber eine halbe Stunde $25 \mathrm{~cm}$ von der Hautoberfläche thätig sein lassen. Dieses Zeitmaass abstrahire ich aus meiner Erfahrıng und der Litteratur, und es ist eher zu niedrig als zn hoch beziffert. Bei der. Haut sehr nahem Rohr sah ich einmal nach einer eine halbe Strunde währenden Exposition eine sehr hartnäckige und schwere Dermatitis auftreten; übrigens die einzige, welche ich in meiner Praxis bei Aufnahmen uberhaupt erlebt habe. - Gerade angesichts der so zahlreichen Mittheilungen von Schäden der Röntgenstrahlen möchte auch ich im Einklang mit den anderen Autoren, welche eine grössere Erfahrung gesammelt haben, hervorheben, dass im Verhältniss zur Zahl der Durchstrahlungen selbst leichte, besonders aber schwere Hautentzündungen sehr selten vorkommen.

Die experimentellen Arbeiten, welche beweisen sollen, dass die $\mathrm{X}$-Strahlen auf das Herz oder die Körpertemperatur einwirken, sind nicht einwandsfrei. Die Fälle von Walsh (Sonnenstich ähnliche Symptome) können anf Zufall beruhen, da sie vereinzelt dastehen.

Die Möglichkeit einer Gefahr von Seiten der X-Strahlen besteht heute jedenfalls nicht mehr bei Untersuchungen, sondern nur bei Experimenten und therapeutischen Anwendungen.

Weil gelegentlich schmerzloser Haarausfall nach Rontgenstrahlen eintrat, hat man bekanntlich die X-Strallen als Depilatorium empfohlen Freund ${ }^{114}$ ) hat einen Naevus pilosus mit mehrore Monate anhaltendem Erfolge bestrahlt. Es wurde dieselbe Stelle der Haut über eine Woche lang täglich ein bis zwei Stunden den Strahlen ausgesetzt. Dies ist aber nicht ungefährlich. Ich habe bei vorsichtigeren Verfahren bisher keine wesentlichen Erfolge erzielen können.

Nach Kümmell ${ }^{18}$ ) und nach Fuch ${ }^{198}$ ) soll Lupus durch X-Strahlen günstig beeinflusst werden, nach Rend ${ }^{12 t}$ ), Fiorentini und Linas chi ${ }^{126}$ ) wurden auch andere Formen der Tuberkulose dadurch gebessert. Bergonié und Mongour ${ }^{125}$ ) bekamen bei Phthisis pulmonum nur zweifelhafte Erfolge.

Dagegen ist zur Controlle der ärztlichen Leistungen die Röntgenkunst in den verschiedensten Gebieten mit Nutzen herangezogen worden.

Was die Sichtbarkeit der X-Strahlen betrifft, so laben mich gemeinsam mit Herrn Cowl ausgefuhrte Untersuchungen gelehrt, dass sie nicht allein von der Intensität der Strahlen abhängt, sondern auch von der Individualität der Versuchspersonen. Unter den gleichen Bedingungen erhalten die einen Lichterscheinungen, die anderen nicht.

In Bezug auf die Einwirkung der X-Strahlen auf Mikroorganismen stimmen die Autoren überein, dass sich eine solche nicht nachweisen lässt.

Alles in allem müssen wir sagen, dass sich Röntgen's Strahlen dieses Jahr ihren Ruf als vortreffliche Hulfe bei der Untersuchung der Kranken bewährt haben und dass sie bei vorsichtiger Handhabung niemals Schaden stiften. $\mathrm{Ob}$ sie aber auch den Ruhm eines Heilmittels beanspruchen können, muss von weiteren Erfahrungen abhängig gemacht werden.

Systematische Litteraturübersicht:

1) Röntgen, Weitere Beobachtungen über die Eigenschaften der $\mathrm{X}$-Strahlen. Sitzungshericht der kőnigl. preuss. Akademie der Wissenschaften zu Berlin, 13. Mai.

Bücher: 2) Büttner und Müller, Technik und Verwerthung der Röntgen'schen Strahlen (für Aerzte). - 3) Rosenfeld, Diagnostik innerer Krankheiten etc. - 4) Parzer-Mühlbacher, Photographische Aufnahmen ..... mittels der Influenzelektrisirnaschine.

Zeitungen: 5) Archives of clinical skiagraphy (London), - - 6) Fortschritte auf dem Gebiete der Röntgenstrahlen, Deycke und AlbersSchönberg (Hamburg). 
Physik und Technik: 7) Laufende Referate in den Beilagen zu den Annalen der Physik. - 8) Grätz, Münch. med. Wochenschr. S. 416 (für Aerzte leicht verständlich).

Methodik: 9) Exner, Wien. klin. Wochenschr. S. 1 (Lokalisation). - 10) Levy-Dorn, Verhandlungen der physiologischen Gesellschaft zu Berlin S. 36 und XXVI. Congress der deutschen Gesellschaft für Chirurgie (idem und Stereoskopie). - 11) Marie und Ribaut, Compt. rend. 124, S. 613 (Stereoskopie). - 12) Cowl, Deutsche med. Wochenschr. S. 265 (Verkürzung der Expositionszeit). - 13) Lev y, XXVI. Congress der deutschen Gesellschaft für Chirurgie (idem). - 14) Hedley, Lancet S. 953 (Form der Platten). - 15) Becher, XV. Congress für innere Medicin (Blutleere).

Uebersichten überVerwerthung in der Medicin: 16) Wagner. Laufende Referate in Schmidt's Jahrbïchern. - 17) Lovy-Dorn, Deutscle med. Wochenschr. No. 8.

Umfassendere Themata: 18) Kummell, Arch. f. klin. Chir. S. 194 (Chirurgie). - 19) Schwertzel, Berl. klin. Wochenschr. S. 628 und 648 (idem). - 20) Wullstein, ibidem S. 334 (Rumpfaufnahmen). 21) Joachimsthal, Therapeutische Monatshefte II und XXVI. Congress der deutschen Gesellschaft für Chirurgie. - 22) Hoffa, ibidem (beide Autoren: Orthopädie). - 23) Grunmach, a) Therapeutische Monatshefte I und b) Wiener med. Wochenschr. S. 1649 (innere Medicin). - 24) Benedikt, XV. Congress für innere Medicin (idem). - 25) Dumstrey, Monatsschrift für Unfallheilkunde No. 11 (Unfälle). - 26) F. de Courmelles, Compt. rend. 124, S. 1179 (idem und Gerichtsmedicin). 27) Brit. med. Journal S. 1126 und 1140 (idem). - 28) Stechow, XXVI. Congress der deutschen Gesellschaft für Chirurgie (Armee). 29) Habert, Wiener klin. Rundschau S. 208 (idem). Vergl. 2 und 18.

Specielles: Fremdkorper: Schädelgegend: 30) Hardt, Münch. med. Wochenschr. S.97. - 31) Péan, Bull. de l'Académ. de méd. S. 286. Vergl. 2 und 18. - Gesicht: 32) Dahlfeld, Dentsche med. Wochenschr. S. 282 (Eisensplitter im Auge). - 33) Brubacher, Mïnch. med. Wochenschr. S. 727. - 34) Benedikt, Wiener klinische Rundschau No. 13 (Kugel im Jochbein). Vergl. 25. - T'on sille siehe 18. - Oshyoid.: 35) Berger, Brit. med. Journ. I, S. 1183 (Nadel bei zweijährigem Kinde). - Oesophagus: 36) Schüller, Berl. klin. Wochenschrift No. 13 (Knochen in der Gegend des Ringknorpels eines Erwachsenen). - 37) Marsh, Lancet S. 313 (Half penny bei zwei Kindern). 38) Bliss, ibidem März („Iron staple“. Erst nach Erkenntniss seiner Form mit der Zange entfernbar). - Thorax: 39) Morris, ibidem No. 6 (Geschoss hinter Clavicula. Operirt). - 40) Lexer, Arch. f. klin. Chir. S. 756 (siehe Text). Vergl. 2 und 34. - Abd omen vergl. 2 und 18. Extremitaten: Viele Berichte zum Theil in den schon angefubiten Arbeiten.

Knochen: Brüche: 41) Jolly, Berl. klin. Wochenschr. S. 215 (Splitterfractur des Schläfenbeins nicht erkannt). - 42) Chipault et Londe, Gazette des hôpit. LXX, 19 (scheineu mehr Glück gehabt zu haben. Beschreiben auch Wirbelfracturen). - Dumstrey siehe No. 25 (Schambeinfractur). - 43) D o n, Brit. med. Journ. S. 797 I (Fract. coll. fem.). - 44) Thiem, XXVI. Congress der deutschen Gesellschaft für Chirurgie (idem). Vergl. auch 18, 19, 20. Ueber Brüche der Extremitäten liegen sehr zahlreiche Mittheilungen vor. - Pseudarthrosen vergl. besonders 19. - Transparenz der Knochen: 45) Espin, Brit. med. Journ. I, S. 799 (bei Tuberkulose). - 46) Göbel, Deutsche med. Wochenschrift S. 267 (Osteomalacie). - 47) $\mathrm{Hahn}$, Wiener med. Club (gegenüber dem Knorpel). - Heerde, Tumoren der Knochen, Osteomyelitis etc.: 48) Müller-Kannberg, Berl. klin. Wochenschr. 15. Februar. - 49) Ullmann, Wien. klin. Wochenschr. S. 164. - 50) Wassermann, ibiderm No. 11. Vergl. 2, 18, 20, 45. - Entwicklung der Knochen: 51) Springer und Serbanesco, Compt. rend. 124, S. 1116. - 52) Behrendsen, Deutsche med. Wochenschr. S. 433. - Plastik und Restitution: 53) Kronacher, Müchener med. Wochenschrift S. 416. - 54) Graff, ibidem S. 786. - 55) Sick, ibidem S. 786 . 56) Ollier, Semaine méd. XVII, 25. - 57) Riedel, XXVI. Congress der deutschen Gesellschaft für Chirurgie. Vgl. 18, 19 etc.

Gelenke: Luxationen: 58) Zenker, Münch. med. Wochenschr. S. 84. - 59) Tausch, ibidem No. 28. - 60) Barabo, ibidem No. 30. 61) Pa is e, Wiener med. Wochenschr. S. 587. Vergl. den allgemeinen Theil. - Ankylosen: 62) Forgue, Revue de chir. No. 9. - 63) Brick, Dentsche med. Wochenschr. S. 152 . Vergl. 21, 48.

Orthopädie: 64) Hofmeister, XXVI. Congress der deutschen Gesellschaft für Chirurgie (Coxa vara). - 65̃) Barwell, Lancet S. 306 (Klumpfuss). - 66) v. Stubenrauch, Münch. med. Wochenschr. S. 696 (Radiusdefect). - 67) Roughton, Lancet 59 (Verkurzung der Metacarpalknochen etc.). Vergl. 21, 22.

Innere Medicin (Knochen): Gicht und Rheumatismus: 68) Totain und Serbanesco, Semaine méd. S. 28. - 69) Spitzer, Deutsche med. Wochenschr. S. 46 (Vereinsblatt). - 70) Rosenfeld, ibidem. - 71) Unna, Münch. med. Wochenschr. S. 431. - 72) Rumpf, XV. Congress fur innere Medicin und Munch. med. Wochenschr. 28. Hypertrophie und Atrophie: 73) Teleky, Wien. klin. Wochenschr S. 143 (Knochenschlägerfinger). - 74) Litten, Berl. klin. Wochenschr.
S. 235 (idem). - 75) Schlesinger, Wien. klin. Rundsch. S. 207 (Osteophyten). - 76) Edel, Berl. klin. Wochenschr. S. 689 (Akromegalie).

Circulationsapparat: Herz und Pericard: 77) Benedikt, Wiener med. Wochenschr. No. 9 und 10 (reichhaltige Casuistik, Vorschlag für Messung der Herzgrösse). - 78) Schott, Deutsche med. Wochenschrift S. 220 (Einfluss von Bad und Gymnastik). - 79) Dumstrey, ibidem S. 287 (Erwiderung auf No. 78). -80 ) Schott. ibidem S. 334 (Antwort auf No. 79). - 81) Berdach, Wiener klin. Wochenschr. 179 (Vitium cordis). - 82) Vehs emeyer. Deutsche med. Wochenschr. S. 180 (Dextrocardie). - 83) Burghardt, ibidem No. 38 (Situs transversus). -84) Sch ott, ibidem S. 495 (Herzuberanstrengung). - 85) B ouchard, Semaine méd. XVII, 25 (bei Aorteninsufficienz deutliche Pulsation der Aorta ascendens und descendens). Vergl. 23, 72. - A orta: 86) Was sermann, Wiener klinische Wochenschr. S. 86. - 87) Galloway. Lancet I, S. 58. - 88) Aron, Deutsche med. Wochenschr. S. 342. - 89) LevyDorn, XV. Congress für innere Medicin. Vergl. 3, 23, 72, 81. - Gefässverkalkungen: siehe $18,19,20,72$.

Respirationstractus: Lungen und Pleura: 90) Martin Durr. Compt. lend. 124, No. 13 (Spitzeninduration). - 91) Bergonié, Semaine méd. S. 6 (Vergleich mit Percussion). Vergl. 3, 23, 24, 72, 86. - Zwerchfell: 92) v. Basch, Wiener med. Wochenschr. S. 185 (geringe Bewegung). - 93) Löbker, XXVI. Congress der deutschen Gesellschaft für Chirurgie (Stillstand). - Gaumensegel: 94) Scheier, Deutsche med. Wochenschr. S. 403 . - Mediastinaltumoren: vergl. $3,23,89$.

Digestionsapparat: Speiserohre: 95) Rumpel, Munch. med. Wochenschr. 15 und 16 (Erweiterung).6- 96) Grab ow er, Verhandlungen der laryngologischen Gesellschaft zu Berlin, Juni (Carcinoma). Vgl. 2, 18. Magen: 97) Linde mann, Deutsche med. Wochenschr. S. 17 (Bestimmung der unteren Grenzen). Vergl. 20. - Darm und grosse Eingeweide: vergl. 2, 3, 18, 20.

Harn- und Geschlechtsapparat: Nieren: 98) Leon, Lancet S. 169 (Stein). - 99) Seiffart, Centralblatt für Gynäkologie No. 1. Vergl. 2, 18, 20, 23. -- Blase: 100) Wolff, Congress der deutschen Gesellschaft für Chirurgie. Vergl. 18, 98. - Steine als Präparat: 101) v. Frisch, Deutsche med. Wochenschr. No. 19. - 102) Buguet und Gascard, Presse médicale 41. - Geburtshülfe: 103) Levy und Thumin, Deutsche med. Wochenschr. S. 507 (Beckenmessung). -104) Levy-Dorn, ibidem 566 (Foetus in vivo; Erwiderung auf 103). 104a) Benedikt, Wiener med. Wochenschr. S. 824.

Schäden: Dermatitis: 105) Thompson, Lancet 195. 106) Ryan, Brit. med. Journ. I, S. 84. - 107) Sor el, Compt. rend. 124, S. 826. - 108) Lannelongue, ibidem S. 828. - 109) Mies, Deutsche med. Wochenschr. No. 26. - 110) Crocker, Brit. med. Journ. I, S. 8. 111) Marcuse, Deutsche med. Wochenschr. No.11. - 112) Apostoli, Compt. rend. 124, S. 1395 (besonders schwerer Fall). - 113) Reid, Scott. med. and surg. Journ. Februar. - Haarausfall: 114) Freund, Wiener klin. Wochenschr. S. 105 und Wiener med. Wochenschr. S. 856. - 115) Forster, Deutsche med. Wochenschr. S. 105. - Andere Schäden: 116) Gilchrist, Bull. of Johns Hopkins Hospit. VIII, S. 71. 117) Seguy und Quénisset, Compt. rend. 124, S. 790 (Einfluss auf Körpertemperatur). - 118) Lecercle, ibidem 125, S. 234. - 119) Walsh, Brit. med. Journ. I, S. 1905 (dem Sonnenstich ähnelnde Wirkung. Magenaffection). - 120) Zuntz und Schumburg, Internationale photographische Monatsschr. für Medicin S. 57 (auf nervöse Centren ohne Einfluss). - Ursache der Schäden: 121) Destot, Compt. rend. 124, S. 1114. - 122) Dale, Med. News S.111. - 123) Thompson, Elektrotechr. Zeitschr. 18, S. 287.

Therapie (ausser für Haarausfall): 124) Rendu, Semaine méd. S. 20 ("Phlegmasie"). - 125) Bergonié et Mongour, Academ. de Méd. 13. Juli (Lungentuberkulose). - 126) Fiorentini und Linaschi, citirt nach Brit. med. Journ. I, S. 1486 (experimentelle Tuberkulose). 127) Immelmann, Deutsche med. Wochenschrift, Therapeutische Beilage S. 61 (Controlle der Pneumatotherapie). - 128) Fuchs, Versammlung deutscher Naturforscher und Aerzte zu Braunschweig (Lupus). Vergl. 18 (idem).

Einfluss auf Mikroorganismen: 129) Sabrazés et Rivière Compt. rend. 124, S. 981. - 130) Sormain, nach Rowland eitirt, Brit. med. Journ. S. 1486. - 132) Blaike, ibidem.

Sichtbarkeit: 133) Brandes und Dorn, Wiedemann's Annalen S. 478. - 134) Cowl und Levy-Dorn, Verhandlungen der physiologischen Gesellschaft zu Berlin S. 55. - 135) D orn, ibidem S. 87 (Erwiderung auf 134). - 136) Cowl und Levy-Dorn, ibidem S. 91 (Antwort auf 135). - 137) Bardet, Compt. rend. 124, S. 1388. Vergl. 1 und 3. 